\title{
Blood Glucose Monitoring and Its Determinants in Diabetic Patients: A Cross-Sectional Study in Shandong, China
}

\author{
Abdul Marouf Raoufi · Xue Tang · Zhengyue Jing • Xinyi Zhang • \\ Qiongqiong $\mathrm{Xu} \cdot$ Chengchao Zhou
}

Received: July 18, 2018 / Published online: September 11, 2018

(C) The Author(s) 2018

\section{ABSTRACT}

Introduction: The aim of this study was to explore the status of blood glucose monitoring and its determinants in diabetic patients in Shandong Province, China.

Methods: This was a cross-sectional survey conducted from 31 August to 12 October 12, in Shandong Province. A multi-stage stratified cluster sampling method was used to select participants. A total of 2183 diabetic patients were included in the analysis. Frequencies and proportions were used to describe the data, and multiple binary logistic regressions were

Abdul Marouf Raoufi and Xue Tang contributed equally to this study and are joint co-first authors of this paper.

Enhanced Digital Features To view enhanced digital features for this article go to https://doi.org/10.6084/ m9.figshare.7021919.

Electronic supplementary material The online version of this article (https://doi.org/10.1007/s13300018-0499-9) contains supplementary material, which is available to authorized users.

A. M. Raoufi · X. Tang · Z. Jing · X. Zhang - Q. Xu School of Public Health, Shandong University, Jinan 250012, China

\section{Zhou ( $\square)$}

School of Public Health, Key Lab of Health

Economics and Policy Research, Shandong

University, Jinan 250012, China

e-mail: zhouchengchao@sdu.edu.cn performed to determine factors associated with blood glucose monitoring.

Results: Of the participants, $51.4 \%$ tested their blood glucose level more than once a month. The multivariate logistic regression model showed that seven variables were significantly associated with the frequency of blood glucose monitoring: education level, residence, household income, self-reported health, physical examination, anti-diabetic drug or insulin injection, and comorbidity.

Conclusions: The level of blood glucose monitoring among diabetic patients in Shandong Province is low. Based on these results we recommend that the cost of blood glucose monitoring by professionals in laboratories be reimbursed or at least reduced and that diabetic patients be encouraged to undergo regular physical examinations.

Keywords: Diabetes; Frequency; Influencing factors; Monitoring of blood glucose

\section{INTRODUCTION}

Diabetes is one of the most important public health problems of the 21st century [1]. The prevalence of diabetes has increased due to changes of lifestyle and an increased life expectancy [2, 3]. According to the International Diabetes Federation, $8.8 \%$ of adults aged 20-79 years were estimated to have diabetes in 
2017, and the number of people with diabetes in that year reached 425 million people worldwide. The number of people with diabetes increases to 451 million if the age range is expanded to 18-99 years. Should these trends continue, by 2045, 693 million people aged 18-99 years, or 629 million people aged 20-79 years, will have diabetes [4]. As in many other countries, the prevalence of diabetes is rising rapidly in China [5]. According to the World Health Organization (WHO) diabetes country profiles for 2016, the number of deaths in China due to diabetes in that year was 224,700 ; in the age range 30-69 years 37,000 of these were male and 56,000 were female, and in the age range $69-70$ years 49,300 were male and 82,400 were female [6].

Blood glucose monitoring can timely reflect the influence of diet, exercise, stress, and drugs on the blood glucose level of patients with diabetes, providing the necessary data for evidenced-based clinical decision-making by healthcare professionals [7]. Several studies have shown that blood glucose monitoring is an effective tool for managing diabetes [8], with regular monitoring of blood glucose levels associated with reduced blood glucose level and an improved quality of life [9-12]. The frequency of blood glucose monitoring negatively correlates with levels of blood glucose [13-17]. Nevertheless, the implementation of blood glucose monitoring is far from satisfactory worldwide [18]. Studies carried out in the USA and Australia found that age, socioeconomic status, and duration of diabetes were associated with compliance with blood glucose monitoring $[14,19,20]$. The authors of two studies carried out in China found that factors affecting the frequency of blood glucose monitoring included diabetes, diet control, exercise therapy, and glycosylated hemoglobin (HbA1c) $[7,17]$. The authors of these studies also found that age, fasting blood sugar, $2 \mathrm{~h}$ postprandial blood sugar, diabetes education, insulin therapy, and hypoglycemia can also affect the frequency of blood glucose monitoring $[7,17]$.

Although there are many health benefits associated with blood glucose monitoring, many diabetics do not test their blood glucose regularly [21]. A patient's blood glucose monitoring compliance is an important factor in determining the performance of blood glucose monitoring, thus it is particularly important to identify a patient's compliance with blood glucose monitoring and the factors that affect it [22]. In China, many patients, especially in rural areas, do not practice self-monitoring of blood glucose (SMBG). Thus, blood glucose levels are monitored by professionals working in laboratories, and the results are of great significance to healthcare professionals who then have trustworthy values on which to base decisions regarding the management of their patient's diabetes. To date, however, most previous studies have focused on SMBG, and few studies have explored blood glucose monitoring by professionals in diabetes patients, especially in China [23-26]. To remedy this situation, the aim of this study was to explore blood glucose monitoring in diabetes patients in China by professionals working in laboratories. To this end, we first explored the prevalence of blood glucose monitoring in diabetes patients by professionals in China, and then we identified the factors associated with blood glucose monitoring.

\section{METHODS}

\section{Subjects}

This study was conducted in Shandong Province, China. Shandong is the second most populated province in China (nearly 100 million in 2016). In 2008, the prevalence of diabetes mellitus in people living in Sandong Province who were aged $\geq 20$ years was $9.9 \%$, which was significantly higher than the average level in the whole country. For our study, we used a multi-stage stratified cluster sampling method to select participants, as described in detail in a previous study [27]. A total of 2481 diabetes patients registered in the NCDs management system in the sampling communities or villages were recruited to the survey, and 2183 diabetic patients for whom a complete dataset were available were included in the analysis, with a response rate of $87.99 \%$. The inclusion criteria were: (1) diagnosis of diabetes 
based on WHO criteria for $>1$ year $[28,29]$; (2) age of $\geq 18$ years; and ( 3 ) ability to understand the questions on the questionnaire.

A cross-sectional study was conducted from August to October 2016. All subjects were interviewed face-to-face using a structured questionnaire by trained Master students from Shandong University School of Public Health. To ensure quality, all of the completed questionnaires were carefully checked by quality supervisors after the interview each day. The questionnaire included: (1) the patient's basic information, such as gender, age, marital status, education, employment status, family size, residence, exercise, household income; (2) patient's health status, including self-reported health, body mass index (BMI), diabetes and its comorbidity, and duration of diagnosis; and (3) health management and health service use, including blood glucose monitoring by professionals, insulin injection, and outpatient and inpatient services.

\section{Variables and Measures}

\section{Dependent Variable}

The dependent variable was the frequency of blood glucose monitoring of participants, and the question was "How often did you monitor your blood glucose by professionals after being diagnosed with diabetes?" The answers were classified into five categories: 1 -more than once a month, 2-once every 1-6 months, 3once every 7-12 months, 4-less than once a year, 5-never. We combined five categories into two categories, namely, monthly and below and more than monthly, in order to conduct binary logistic regression, according to the Guideline for Blood Glucose Monitoring in China [7]. In the present study, "monitoring by professionals" refers to blood glucose being measured during visits to a healthcare professional.

\section{Independent Variable}

Independent variables included socio-demographic characteristics, such as gender (male vs. female), age ( $\leq 60,61-70,>70$ years), marital status (single or married, with single encompassing the not-married, divorced, and widowed states, respectively), education level (illiterate, primary school, junior school, high school and higher), employment status (unemployed vs. employed), family size ( $\leq 3$ vs. $>3$ ), residence (rural vs. urban), exercise (no vs. yes), and household income (Q1, Q2, Q3, and Q4 quintiles, whereby quintile $1(\mathrm{Q} 1)$ was the lowest income and quintile 4 (Q4) was the highest income. Also included as independent variables were self-reported health (good, normal, poor), physical examination (no vs. yes, whereby physical examination means regular physical examination [excluding check-ups for the treatment of diseases]), BMI $(<18.5$, $\left.18.5-23.99,24-27.99, \geq 28 \mathrm{~kg} / \mathrm{m}^{2}\right)$, duration of diagnosis $(\leq 5,6-10,>10$ years), anti-diabetic drug or insulin therapy in the past 2 weeks (no vs. yes), diabetes-related education (no vs. yes), outpatient service in the past 2 weeks (no vs. yes), inpatient service in the past year (no vs. yes), and comorbidity (no vs. yes). In this study, comorbidity means the presence of any other disease(s) or condition(s) (diabetic nephropathy, diabetic eye complications, diabetic foot, diabetic cardiovascular complications, diabetic cerebrovascular disease, and diabetic neuropathy). The measurement details and the code for each variable are presented in Electronic Supplementary Material (ESM) 1.

\section{Statistical Analysis}

Data was entered into a computer using the Statistical Package for Social Sciences (SPSS) software version 24.0 (IBM Corp., Armonk, NY, USA). Data was described using frequencies and proportions wherever appropriate. Univariate logistic regression analysis was used to analyze the effect of each potential factor on a patient's blood glucose monitoring compliance. Multiple binary logistic regressions were performed to determine variables for which a $P$ value of $<0.05$ in univariate logistic regression were associated with blood glucose monitoring adherence. A $P$ value of $<0.05$ was considered to be statistically significant. Survey procedures were used to analyze survey data by taking into account the sample design. 


\section{Compliance with Ethics Guidelines}

The study protocol for this study involving human participants was approved by the Ethical Committee of Shandong University School of Public Health and was in accordance with the 1964 Helsinki declaration and its later amendments or comparable ethical standards. The investigation was conducted after the informed consent of all participants was obtained.

\section{RESULTS}

\section{Patients' Characteristics}

A total of 2183 patients with DM (1461 females, 722 males) were enrolled in this study. Their age ranged from 21 to 106 years, and the majority $(85.0 \%)$ were married. With respect to education level, $7.2 \%$ of the participants had high school and above,, $18.3 \%$ had junior school education, $30.7 \%$ had primary school education, and $43.7 \%$ had no formal education. Half of the participants $(50.0 \%)$ were unemployed, and the majority (86.3\%) had a family size of $\leq 3$ people. By far the majority (88.2\%) lived in rural areas, with only $11.8 \%$ living in urban areas. Only $22.5 \%$ of the participants thought they had a good health condition, although $62.1 \%$ of all participants reported exercising regularly. The majority $(88.4 \%)$ of the participants had had the physical examination. The BMI ranged between 18.5 and $23.99 \mathrm{~kg} / \mathrm{m}^{2}$ for $31.4 \%$ of participants, and $66.0 \%$ of participants were overweight. Overall, $29.7 \%$ of patients had diabetes for $>10$ years, and $91.3 \%$ of participants had used anti-diabetic drug or injected insulin in the past 2 weeks. The percentages of anti-diabetic drug or insulin injection are given in ESM 2. Only $14.0 \%$ of participants had no diabetes-related education, $17.4 \%$ had diabetes complications, $2.7 \%$ of used outpatient service, and $7.7 \%$ used inpatient service (Table 1 ).
Table 1 Basic information on 2183 participants with diabetes mellitus in Shandong, China, 2016

\begin{tabular}{|c|c|c|}
\hline Characteristics & $N$ & Weight (\%) \\
\hline \multicolumn{3}{|l|}{ Gender $(n)$} \\
\hline Male & 722 & 33.1 \\
\hline Female & 1461 & 66.9 \\
\hline \multicolumn{3}{|l|}{ Age (years) } \\
\hline$\leq 60$ & 554 & 28.3 \\
\hline $61-70$ & 1002 & 45.5 \\
\hline$>70$ & 627 & 26.2 \\
\hline \multicolumn{3}{|l|}{ Marital status } \\
\hline Single ${ }^{a}$ & 327 & 15.0 \\
\hline Married & 1856 & 85.0 \\
\hline \multicolumn{3}{|l|}{ Education } \\
\hline Illiterate & 826 & 43.7 \\
\hline Primary school & 732 & 30.7 \\
\hline Junior school & 449 & 18.3 \\
\hline High school and above & 176 & 7.2 \\
\hline \multicolumn{3}{|l|}{ Employment status } \\
\hline Unemployed & 1195 & 50.0 \\
\hline Employed & 988 & 50.0 \\
\hline \multicolumn{3}{|l|}{ Family size } \\
\hline$\leq 3$ & 1900 & 86.3 \\
\hline$>3$ & 283 & 13.7 \\
\hline \multicolumn{3}{|l|}{ Residence } \\
\hline Rural & 1692 & 88.2 \\
\hline Urban & 491 & 11.8 \\
\hline \multicolumn{3}{|l|}{ Exercise } \\
\hline No & 808 & 37.9 \\
\hline Yes & 1375 & 62.1 \\
\hline \multicolumn{3}{|l|}{ Household income ${ }^{b}$} \\
\hline Q1 & 546 & 28.8 \\
\hline Q2 & 549 & 25.7 \\
\hline Q3 & 543 & 25.2 \\
\hline Q4 & 545 & 20.3 \\
\hline
\end{tabular}


Table 1 continued

\begin{tabular}{lll}
\hline Characteristics & $N$ & Weight (\%) \\
\hline
\end{tabular}

Self-reported health

Good 521

22.5

Normal

913

42.4

Poor

749

35.2

Physical examination

$$
\text { No }
$$

Yes

1909

88.4

Body mass index $\left(\mathrm{kg} / \mathrm{m}^{2}\right)$

$<18.5$

18.5-23.99

31.4

24-27.99

954

43.6

$\geq 28$

504

Duration of diagnosis (years)

$\leq 5$

38.3

6-10

680

32.0

$>10$

29.7

Type of diabetes

I

11.3

II

1948
Table 1 continued

\begin{tabular}{lcc}
\hline Characteristics & $\boldsymbol{N}$ & Weight (\%) \\
\hline No & 2012 & 92.3 \\
Yes & 171 & 7.7 \\
\hline
\end{tabular}

a Single includes not married, divorced, and widowed

b Quintile 1 (Q1) was the lowest income and Q4 was the highest income, with Q3 bing a medium-level income

\section{The Frequency of Blood Glucose Monitoring}

Of the total 2183 participants, 51.4 and $48.6 \%$ had their blood monitored by professionals more than once a month and once or less than once a month, respectively. In the latter category, $41.0 \%$ had tested their blood glucose more than once every 6 months and $4.7 \%$ had their blood glucose level tested once or less than once every 6 months. Of the participants, 1.9\% had tested their blood glucose less than once a year and $1.0 \%$ had never tested their blood glucose (Table 2).

\section{Factors Associated with Frequency of Blood Glucose Monitoring}

Anti-diabetic drug or insulin injections

$\begin{array}{lrr}\text { No } & 174 & 8.7 \\ \text { Yes } & 2009 & 91.3\end{array}$

Diabetes-related education

$\begin{array}{lrr}\text { No } & 317 & 14.0 \\ \text { Yes } & 1866 & 86.0\end{array}$

Comorbidity

$\begin{array}{lrr}\text { No } & 1796 & 82.6 \\ \text { Yes } & 387 & 17.4\end{array}$

Outpatient service

$\begin{array}{rrr}\text { No } & 2121 & 97.3 \\ \text { Yes } & 62 & 2.7\end{array}$

Inpatient service
The results of the univariate and multivariate analyses are shown in Table 3. In the multivariate analysis, we analyzed the 18 factors itemized in section "Independent variable"; of these, 11 were associated with blood glucose monitoring in the univariate analysis. The only variables not significantly associated with blood glucose monitoring were employment status, exercise, disease duration, and diabetes-related education $(P>0.05)$. Participants who had a higher education $(P<0.001)$, lived in urban areas (odd ratio [OR] 2.83; 95\% confidence interval $[\mathrm{CI}] 1.75,4.57)$, had a higher household income $(P<0.05)$, had a poor or normal selfreported health condition $(P<0.05)$, had physical examinations (OR 1.56; 95\% CI 1.06, $2.31)$, took an anti-diabetic drug or had injected insulin in the past 2 weeks (OR 2.95; 95\% CI 
Table 2 The frequency of blood glucose monitoring by professionals in Shandong, China, 2016

\begin{tabular}{lrc}
\hline Frequency & $\boldsymbol{N}$ & $\begin{array}{l}\text { Percentage of } \\
\text { participants }\end{array}$ \\
\hline More than once a month & 1197 & 51.4 \\
More than once every & 819 & 41.0 \\
$\quad 6$ months & & \\
Once every 6 months or & 98 & 4.7 \\
$\quad$ less & & \\
Less than once a year & 43 & 1.9 \\
Never & 26 & 1.0 \\
Total & 2181 & 100.0 \\
\hline
\end{tabular}

$1.38,6.30)$, and had diabetic comorbidity (OR 1.28; 95\% CI 1.06, 1.69) had more often monitored their blood glucose compared to their counterparts.

\section{DISCUSSION}

With the emergence of such social phenomena as rapid economic development, urbanization, an increasingly aged population, and changes in people's lifestyle, diabetes has become a major chronic disease that threatens human health worldwide [30, 31]. The prevalence of diabetes has increased rapidly during the past decades [32]. Previous studies have shown that an increased monitoring frequency is associated with a decrease in blood glucose level, and the association between self-monitoring frequency and glycemic control has been definitely confirmed in patients with diabetes [22]. Blood glucose monitoring is therefore indispensable in any comprehensive management program aimed at the prevention and treatment of diabetes.

According to the 2015 edition of the Guidelines for Blood Glucose Monitoring in China [7], people who use lifestyle intervention or oral hypoglycemic drugs to manage their diabetes should monitor their blood glucose at least twice a week on average. In our study we focused only on blood glucose monitoring performed in professional laboratories. Many diabetic patients, especially in rural areas, do not practice SMBG. On the other hand, SMBG can only improve a patient's blood glucose level if the patient is able to measure his/her blood glucose accurately, interpret the measurements, and then make the correct decision based on these values. Thus, the monitoring of blood glucose levels in laboratories by professionals is also necessary because the professionals can provide advice on controlling blood glucose and also assist with the interpretation of results. Previous studies focused on the SMBG, such as the study of Tingting et al. showed that the average monthly self-glycemic monitoring frequency was 10.03 times among the 289 patients surveyed [7]. Skelly and colleagues showed that $76.7 \%$ of patients tested their blood glucose at least once a week [14]. However, we did not find similar research involving the frequency of blood glucose monitoring in laboratories. Our study showed that only $54.8 \%$ of the participants had tested their blood glucose more than once per month, indicating that blood glucose monitoring of diabetic patients in Shandong Province is at a low level.

The results of our study showed that the frequency of blood glucose monitoring was lower in illiterate participants than in those with a high school education and above; these findings are consistent with those of Adams et al. [19]. It is possible that patients with a relatively lower education may have difficulty in understanding both the importance of blood glucose monitoring and how to self-monitor. Equally, a low education may lead to a low income. This finding implies a need for physicians to pay more attention to glucose monitoring in diabetes patients with a lower education.

We found that the frequency of blood glucose monitoring was significantly lower among participants from rural areas than among those from urban areas. This finding suggests that the difference in socioeconomic development and quality of healthcare services between rural and urban areas may explain the observed difference in frequency of blood glucose monitoring. Another possible explanation is that the annual household income and diabetes-related education in the urban diabetic patients of our study 
Table 3 Logistic regression analysis of factors affecting patients' blood glucose monitoring by professionals in Shandong, China, 2016

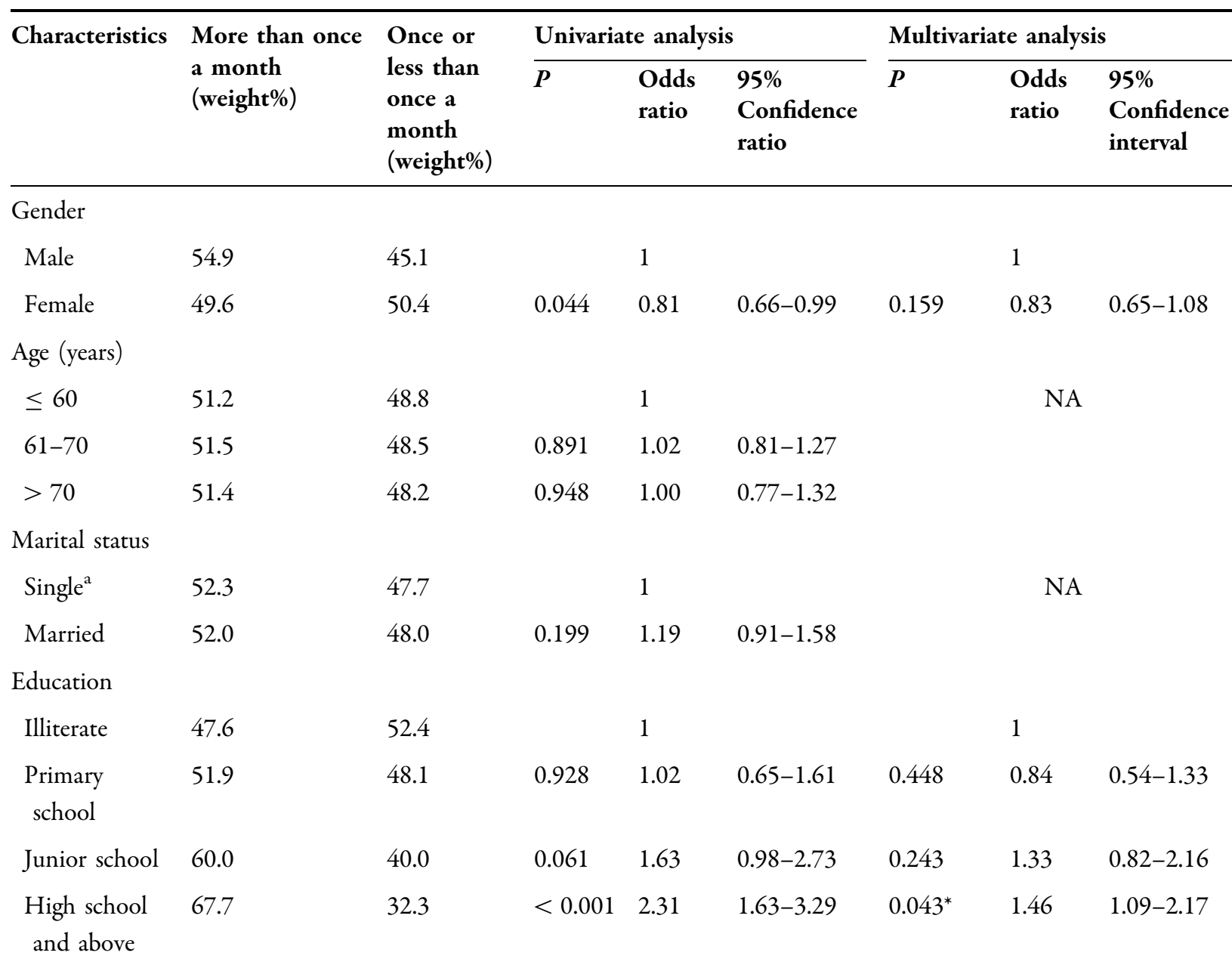

Employment status

\section{Unemployed 55.9}

Employed $\quad 46.8$

Family size

$$
\leq 3 \quad 50.5
$$$$
>3
$$$$
57.2
$$

49.5

42.8

Residence

$\begin{array}{ll}\text { Rural } & 47.8 \\ \text { Urban } & 78.3\end{array}$

Household income ${ }^{\mathrm{b}}$

\begin{tabular}{ll} 
Q1 & 43.9 \\
Q2 & 48.9 \\
Q3 & 51.4 \\
\hline
\end{tabular}

52.2

21.7$$
1
$$$$
0.017 \quad 0.69
$$$$
0.52-0.93
$$$$
0.118
$$

1

$0.80 \quad 0.61-1.06$
1 NA

$0.221 \quad 1.31 \quad 0.84-2.04$ 
Table 3 continued

\begin{tabular}{|c|c|c|c|c|c|c|c|c|}
\hline \multirow[t]{2}{*}{ Characteristics } & \multirow{2}{*}{$\begin{array}{l}\text { More than once } \\
\text { a month } \\
(\text { weight } \%)\end{array}$} & \multirow{2}{*}{$\begin{array}{l}\text { Once or } \\
\text { less than } \\
\text { once a } \\
\text { month } \\
\text { (weight\%) }\end{array}$} & \multicolumn{3}{|c|}{ Univariate analysis } & \multicolumn{3}{|c|}{ Multivariate analysis } \\
\hline & & & $\bar{P}$ & $\begin{array}{l}\text { Odds } \\
\text { ratio }\end{array}$ & $\begin{array}{l}95 \% \\
\text { Confidence } \\
\text { ratio }\end{array}$ & $\bar{P}$ & $\begin{array}{l}\text { Odds } \\
\text { ratio }\end{array}$ & $\begin{array}{l}95 \% \\
\text { Confidence } \\
\text { interval }\end{array}$ \\
\hline Q4 & 65.2 & 34.8 & 0.001 & 2.39 & $1.51-3.79$ & $0.019^{*}$ & 1.54 & $1.08-2.17$ \\
\hline
\end{tabular}

Exercise

$\begin{array}{llllll}\text { No } & 46.2 & 53.8 & & 1 & \\ \text { Yes } & 47.5 & 52.5 & 0.077 & 1.29 & 0.97-1.71\end{array}$

Self-reported health

$\begin{array}{ll}\text { Good } & 57.6 \\ \text { Ordinary } & 49.4 \\ \text { Poor } & 49.8\end{array}$

42.4

50.6

$0.001 \quad 0.72$

$0.59-0.87$

$0.019^{*}$

1

50.2

$0.067 \quad 0.73$

$0.52-1.02 \quad 0.040^{*}$

0.72

(0.55-0.94)

Physical examination

$\begin{array}{ll}\text { No } & 40.7 \\ \text { Yes } & 52.8\end{array}$

59.3

47.2

1
$0.017 \quad 1.63$

$\begin{array}{lll}1.10-2.42 \quad 0.026^{*} & 1.56\end{array}$

$1.06-2.31$

Body mass index

$\begin{array}{ll}<18.5 & 39.6 \\ 18.5-23.99 & 46.3 \\ 24-27.99 & 54.1 \\ \geq 28 & 54.5\end{array}$

60.4

53.7

45.9

45.5

$0.017-1.63$

(1.

NA

Duration of diagnosis (years)

$\begin{array}{ll}\leq 5 & 47.0 \\ 6-10 & 51.3 \\ >10 & 57.2\end{array}$

53.0

48.7

42.8

0.149

1

0.363

1.32

$0.71-2.42$

0.425

Type of diabetes

$\begin{array}{ll}\text { I } & 23.9 \\ \text { II } & 54.0\end{array}$

76.1

$0.038 \quad 1.80$

1.04-3.13 0.065

$1.28 \quad 0.68-2.40$

$0.052 \quad 1.83$

0.99-336 $\quad 0.064$

1.68

$0.97-2.91$

Insulin injections

$\begin{array}{ll}\text { No } & 23.9 \\ \text { Yes } & 54.0\end{array}$

76.1

0.034

1

1

$\begin{array}{lll}1.19 & 0.94-1.52 & 0.432\end{array}$

$1.110 .85-1.46$

$\begin{array}{ll}7.2 & 42.8\end{array}$

1.51

1.03-2.19

0.260

$1.23 \quad 0.85-1.78$

Diabetes-related education

$\begin{array}{ll}\text { No } & 32.1 \\ \text { Yes } & 54.5\end{array}$

Comorbidity

67.9

45.5

$\begin{array}{ccc} & 1 \\ 0.900 & 1.02 & 0.74-1.41\end{array}$

NA

46.0

46.0

$<0.001 \quad 3.73$

$2.33-5.97$

$0.007^{*}$

$2.95 \quad 1.38-6.30$

Comorbidity 
Table 3 continued

\begin{tabular}{|c|c|c|c|c|c|c|c|c|}
\hline \multirow[t]{2}{*}{ Characteristics } & \multirow{2}{*}{$\begin{array}{l}\text { More than once } \\
\text { a month } \\
(\text { weight } \%)\end{array}$} & \multirow{2}{*}{$\begin{array}{l}\text { Once or } \\
\text { less than } \\
\text { once a } \\
\text { month } \\
\text { (weight\%) }\end{array}$} & \multicolumn{3}{|c|}{ Univariate analysis } & \multicolumn{3}{|c|}{ Multivariate analysis } \\
\hline & & & $\bar{P}$ & $\begin{array}{l}\text { Odds } \\
\text { ratio }\end{array}$ & $\begin{array}{l}95 \% \\
\text { Confidence } \\
\text { ratio }\end{array}$ & $\bar{P}$ & $\begin{array}{l}\text { Odds } \\
\text { ratio }\end{array}$ & $\begin{array}{l}95 \% \\
\text { Confidence } \\
\text { interval }\end{array}$ \\
\hline No & 50.0 & 50.0 & & 1 & & & 1 & \\
\hline Yes & 58.6 & 41.4 & 0.020 & 1.42 & $1.06-1.91$ & $0.045^{*}$ & 1.28 & $1.06-1.69$ \\
\hline \multicolumn{9}{|c|}{ Outpatient service } \\
\hline No & 51.3 & 48.7 & & 1 & & NA & & \\
\hline Yes & 53.4 & 46.6 & 0.815 & 1.09 & $0.53-2.22$ & & & \\
\hline \multicolumn{9}{|l|}{ Inpatient service } \\
\hline No & 50.8 & 49.2 & & 1 & & & 1 & \\
\hline Yes & 58.1 & 41.9 & 0.014 & 1.34 & $1.06-1.68$ & 0.160 & 1.22 & $0.92-1.61$ \\
\hline
\end{tabular}

*Significantly associated with blood glucose monitoring at $P<0.05$

$N A$ Not applicable

a Single includes not married, divorced, and widowed

b Quintiles as defined in Table 1

were higher than those in rural patients. In China, the quality of healthcare in urban areas is far above that in rural regions. Therefore, we should improve the quality of the healthcare services in rural regions along with providing more diabetes-related education for diabetic patients in rural regions.

Consistent with the finding of Yuan et al., our study also shows that economic status is the most significant factor affecting blood glucose monitoring of diabetes in Shandong Province [22]. In China, the cost of monitoring blood glucose and of acquiring a glucose meter and strips for diabetes is largely paid for by the patients themselves, leading to many patients not conducting regular blood glucose monitoring. Therefore, we hope that the national health insurance agency will reimburse the cost of monitoring blood glucose in laboratories and the acquisition of a glucose meter and strips with the aim to reduce the economic burden of diabetes.

In our survey, participants with frequent physical examinations had monitored their blood glucose level more often than those with less frequent physical examinations. People who undergo frequent physical examinations will be better able to recognize the significance of blood glucose levels and identify abnormal levels early. We recommend that the government provide more publicity and education about the advantages of frequent physical examinations.

Participants with a diabetes duration of $>10$ years monitored their blood glucose more often than those with a disease duration of $\leq 5$ years. Interestingly, multivariate analysis of the logistic regression showed that disease duration was not associated with the frequency of blood glucose monitoring to any great degree. We found that diabetes patients with complications monitored their blood glucose more often than patients with no complications. One explanation is that patients with longer disease duration may have more complications, so they may pay more attention to blood glucose monitoring.

There are a number of limitations to this study. First, this study was cross-sectional, and we could not directly assess cause-and-effect 
relationships of demographic and disease characteristics with the frequency of monitoring or adherence. Second, the age distribution of our study was uneven, and most of the participants were elderly, which may have introduced bias into the results. Finally, in this study we focused on blood glucose monitoring in laboratories by professionals and did not take the SMBG into account, which will be remedied in our followup study.

\section{CONCLUSIONS}

The results of our study show that the level of blood glucose monitoring is still low in diabetic patients in Shandong Province in China. The patients who have a relatively lower education background were found to be less likely to be adherent, which implies a need for physicians to pay more attention to the monitoring in such patients. The government should reimburse the cost of monitoring blood glucose in laboratories, blood glucose meters, and strips, and encourage regular physical examinations.

\section{ACKNOWLEDGEMENTS}

We thank the officials of local health agencies and all participants and staff at the study sites for their cooperation.

Funding. This research was supported by the National Natural Science Foundation of China (7100306, 71473152 and 71774104), Cheeloo Young Scholar Grant, and Shandong University (IFYT1810, 2012DX006). The funding agencies played no role in the design, analysis, and writing of the article, nor in the journal processing charges. The article processing charges were funded by the authors.

Authorship. All named authors meet the International Committee of Medical Journal Editors (ICMJE) criteria for authorship for this article, take responsibility for the integrity of the work as a whole, and have given their approval for this version to be published.
Authorship Contributions. Chengchao Zhou conceived the idea and provided the finishing touches to the manuscript. Abdul Marouf Raoufi and Xue Tang coded and analyzed the data and wrote the manuscript. Zhengyue Jing, Xinyi Zhang and Qiongqiong Xu participated in the research design and data collection.

Disclosures. Abdul Marouf Raoufi, Xue Tang, Zhengyue Jing, Xinyi Zhang, Qiongqiong $\mathrm{Xu}$ and Chengchao Zhou have nothing to disclose.

Compliance with Ethics Guidelines. The study protocol for this study involving human participants was approved by the Ethical Committee of Shandong University School of Public Health and was in accordance with the 1964 Helsinki declaration and its later amendments or comparable ethical standards. The investigation was conducted after the informed consent of all participants was obtained.

Data Availability. Please contact the corresponding author for data requests.

Open Access. This article is distributed under the terms of the Creative Commons Attribution-NonCommercial 4.0 International License (http://creativecommons.org/licenses/ by-nc/4.0/), which permits any noncommercial use, distribution, and reproduction in any medium, provided you give appropriate credit to the original author(s) and the source, provide a link to the Creative Commons license, and indicate if changes were made.

\section{REFERENCES}

1. International Diabetes Federation. The global burden (2005). http://www.idf.org/diabetesatlas/5e/ the-global-burden.

2. Werfalli M, Engel ME, Musekiwa A, et al. The prevalence of type 2 diabetes among older people in Africa: a systematic review. Lancet Diabetes Endocrinol. 2016;4(1):72-84.

3. $\mathrm{Xu} \mathrm{Y,} \mathrm{Wang} \mathrm{L,} \mathrm{He} \mathrm{J,} \mathrm{et} \mathrm{al.} \mathrm{Prevalence} \mathrm{and} \mathrm{control} \mathrm{of}$ diabetes in Chinese adults. JAMA. 2013;310(9): 948-58. 
4. International Diabetes Federation. Diabetes atlas. 8th edn. (2017). http://www.diabetesatlas.org.

5. Shen J, Goyal A, Sperling L. The emerging epidemic of obesity, diabetes, and the metabolic syndrome in china. Cardiol Res Pract. 2012;2012(19):178675.

6. World Health Organization. Diabetes country profiles 2016: China. http://www.who.int/diabetes/ country-profiles/chn_en.pdf?ua=1.

7. Tingting G, Jianshuang $Z$, et al. Status and influencing factors of self-monitoring blood glucose in patients with type 2 of diabetes mellitus. Clin Focus. 2017;32(4):317-22 (in Chinese).

8. Pan C, Yang W, Jia W, et al. Management of Chinese patients with type 2 diabetes, 1998-2006: the Diabcare-China surveys. Curr Med Res Opin. 2009;25(1):39-45.

9. Murata GH, Shah JH, Hoffman RM, et al. Intensified blood glucose monitoring improves glycemic control in stable, insulin-treated veterans with type 2 diabetes: the Diabetes Outcomes in Veterans Study (DOVES). Diabetes Care. 2003;26(6):1759-63.

10. Chidum E, Agbai D, et al. Self-monitoring of blood glucose improved glycaemic control and 10-year coronary heart disease risk profile of type 2 diabetic patients. Chin Med J. 2011;124(2):166.

11. Polonsky WH, Fisher L, Schikman CH, et al. Structured self-monitoring of blood glucose significantly reduces $\mathrm{A} 1 \mathrm{C}$ levels in poorly controlled, noninsulin-treated type 2 diabetes: results from the structured testing program study. Diabetes Care. 2011;34(5):e58.

12. Schneider B, Martin S, Heinemann L, et al. Interrelations between diabetes therapy, self-monitoring of blood glucose, blood glucose and non-fatal or fatal endpoints in patients with type 2 diabetes/results of a longitudinal cohort study (ROSSO 5). Arzneimittel-Forschung. 2007;57(12):762-9.

13. Aydın H, Deyneli O, Yavuz D, Tarçın Ö, Akalın S. Does the frequency of the self-monitoring of blood glucose influence glycemic control in type 2 diabetic patients? Marmara Med J. 2005;1:13-6.

14. Skelly AH, Arcury TA, Snively BM, et al. Self-monitoring of blood glucose in a multiethnic population of rural older adults with diabetes. Diabetes Educ. 2005;31(1):84-90.

15. Schwedes U, Siebolds M, Mertes G. Meal-related structured self-monitoring of blood glucose effect on diabetes control in non-insulin-treated type 2 diabetic patients. Diabetes Care. 2002;25(11):1928-32.
16. Barnett AH, Krentz AJ, Strojek K, et al. The efficacy of self-monitoring of blood glucose in the management of patients with type 2 diabetes treated with a gliclazide modified release-based regimen. A multicentre, randomized, parallel-group, 6-month evaluation (DINAMIC 1 study). Diabetes Obes Metab. 2010;10(12):1239-47.

17. Chai SJ, Jia WN. Analysis of blood glucose monitoring frequency and influencing factors in outpatients with diabetes (in Chinese). Jiangsu Med J. 2007;33(12):1298.

18. Karter AJ. The role of self-monitoring of blood glucose in glycemic control. Endocr Pract. 2006;12[Suppl 1]:110-7.

19. Adams AS, Mah C, Soumerai SB, et al. Barriers to self-monitoring of blood glucose among adults with diabetes in an HMO: a cross sectional study. BMC Health Serv Res. 2003;3(1):6.

20. Bruce DG, Davis WA, Cull CA, et al. Diabetes education and knowledge in patients with type 2 diabetes from the community: the Fremantle Diabetes Study. J Diabetes Complicat. 2003;17(2):82-9.

21. Hu ZD, Zhang KP, Huang Y, Zhu S. Compliance to self-monitoring of blood glucose among patients with type 2 diabetes mellitus and its influential factors: a real-world cross-sectional study based on the Tencent TDF-I blood glucose monitoring platform. Mhealth. 2017;3:25.

22. Yuan L, Guo X, Xiong Z, et al. Self-monitoring of blood glucose in type 2 diabetic patients in China: current status and influential factors. J Chin Med J. 2014;127(2):201-7.

23. Sun X, Huang X, Yuan C, Cui Y. The relationship between coping strategy and self-management behavior among patients with type 2 diabetes. J Nurs Train. 2012;27:1084-6 (In Chinese).

24. Guo XH, Yuan L, Lou QQ, et al. A nationwide survey of diabetes education, self-management and glycemic control in patients with type 2 diabetes in China. Chin Med J. 2012;125:4175-80.

25. Xu Y, Toobert D, Savage C, Pan W, Whitmer K. Factors influencing diabetes self-management in Chinese people with type 2 diabetes. Res Nurs Health. 2008;31:613-25.

26. Huang J, Liu Y, Zhang Y, Yao H. Correlation between self-management and knowledge of and attitude to diabetes in type 2 diabetic patients in Changsha. J Central South Univ. 2013;38:176-81.

27. Jing Z, Chu J, Imam Syeda Z, et al. Catastrophic health expenditure among type 2 diabetes mellitus patients: a province-wide study in Shandong, 
China. J Diabetes Investig. 2018. https://doi.org/10. 1111/jdi.12901.

28. Alberti KG, Zimmet PZ. Definition, diagnosis and classification of diabetes mellitus and its complications. Part 1: diagnosis and classification of diabetes mellitus provisional report of a who consultation. Diabet Med. 2015;15(7):539-53.

29. Xu Y, Toobert D, Savage C, et al. Factors influencing diabetes self-management in Chinese people with type 2 diabetes. Res Nurs Health. 2010;31(6): 613-25.
30. Gong P, Liang S, Carlton EJ, et al. Urbanisation and health in China. Lancet. 2012;379(9818):843-52.

31. Hu FB. Globalization of diabetes: the role of diet, lifestyle, and genes. Diabetes Care. 2011;34(6): 1249-57.

32. Wild S, Roglic G, Green A, Sicree R, King H. Global prevalence of diabetes: estimates for the year 2000 and projections for 2030. Diabetes Care. 2004;27(5):1047-53. 